\title{
Intelligent Tour Planning System using Crowd Sourced Data
}

\author{
Md. Saef Ullah Miah ${ }^{\mathrm{a}}$, Md. Masuduzzaman ${ }^{\mathrm{a}}$, Williyam Sarkar ${ }^{\mathrm{a}}, \mathrm{H}$ M Mohidul Islam ${ }^{\mathrm{a}}$, Faisal \\ Porag $^{\mathrm{a}}$, Sajjad Hossain ${ }^{\mathrm{a}}$ \\ ${ }^{a}$ Dept. of Computer Science and Engineering, American International University-Bangladesh, Dhaka- \\ 1213, Bangladesh
}

Received: 22 October 2017; Accepted: 19 November 2017; Published: 08 January 2018

\begin{abstract}
To observe the beauty of nature and to visit various places around the world, a vast number of tourists visit different countries and many tourist attraction sites now-a-days. But Most of the tourist places have failed to introduce itself as a tourist destination to the visitor due to lack of proper information and proper guideline to visit there. This paper tries to focus on some problems in the tourism industry and try to solve those problems using crowd sourced data with some customized algorithms. Some of the main problems are the lack of information about a destination tourist spot, combination on budget to visit the spot, time of travels etc. We proposed a customize algorithm which will provide maximum suggestion to visit a place with nearest all sub place based on user destination within their given budget and time. Using our method, user can choose the most suitable plan for them to visit those places.
\end{abstract}

Index Terms: Data Crowdsourcing, Tourist, BFS, DFS, Dijkstra, Tour Planner, Route Suggestion.

(C) 2018 Published by MECS Publisher. Selection and/or peer review under responsibility of the Research Association of Modern Education and Computer Science.

\section{Introduction}

Crowdsourcing is known as the operation of snatching job or capitalization. From a group or crowd of people, crowdsourcing is usually done by online or offline. Innovation, problem solving, or efficiency are the common intention for engaging a 'crowd' or group of people is the main practice of it. In general crowdsourcing is a process of work done by a crowd of people. In tourism crowd sourcing is intended to perform the collaborative updating of the tourism resources knowledge base system considering the tourists and tourism businesses [1]. Gathering more and more information is the base of a tourism system. Every country of this

* Corresponding author.

E-mail address: saef@aiub.edu, masud.prince@aiub.edu, williyam.sarkar@gmail.com, mislam6789@gmail.com, 
world has been taken this issue seriously so that this information can be easily increased and developed day by day [2].

We tried to find out some widespread problem in the tourism sector. Inevitably the tourism industry is also affected by the information technological revolution. According to research paper of strategic use of information technologies in the tourism industry [8], tourism is inevitably influenced by the business process re-engineering experienced due to the technological revolution. The impact of information technologies on businesses over $60 \%$. On other research of Rich Harrill et al. [3] Tourists become sophisticated and more demanding to constitute their tour outline easier. A map with regional attraction is potentially useful in terms of attracting tourist to remote areas and this could counteract effort to reduce the distance of travel [4]. Therefore, effective tourism marketing requires [5]. Sharing information through technology can create a phenomenon to receive adequate attention in tourism. A successful tour depends on planning in most of the cases. Moreover, planning is blamed as a failure if proper plan implementation is interrupted anyhow [7]. Tourist also wanted to make their tour successful within their desired budget. But budget and time always conflict with each and others [6]. If tourist can get a proper idea or suggestion considering their plan, this will give comfort to millions of people in this world. Even later they can share their tour information with others so that everyone might get some idea before planning a tour.

A few researchers have investigated the relationship between urban space and attitudes toward tourism development. They also try to generate connections between tourism zones and the physical distance between residents and tourists in specific residential area. Based on the variables of size, distance and location, the attachment gets weaker as the population and density increase [8]. Tourism has become an extremely dynamic system. Economic globalization, fast changing customer behavior, development of transport system and information technologies, modifications in the forms of organizations and labor, all of them are strongly influence the industry. In this scenario, the intensified marketing efforts of all tourism organizations have led to a more effective and positive approach. [9]

\section{Related Work}

However to get the solution, first we analysis some research paper of DFS algorithm [10][11][12]. The depth first search algorithm is a recursive algorithm which uses the idea of backtracking. If we consider all node as a location then DFS can visit of all the location by going ahead, if there are no more location available then it normally searches by backtracking. All the possible location will be visited on the current path till all the unvisited location have been traversed. But the problem of DFS is, it normally searches by moving forward but not by considering cost. We also analysis breadth first search algorithm [14] and heuristic search [13] algorithm to get out expected result. To get the optimized cost base solution, we analysis Dijkstra's Algorithm [14]. Dijkstra algorithms generally follows Greedy approach. It can find the minimum cost required to visit from one location to another location. But getting final cost visiting all location and return to source is not possible by Dijkstra algorithms. Dijkstra uses a priority queue, implemented with a heap.

Then we analysis Brute force algorithm. To get the best combination we try to use brute force algorithm. Because it consists of systematically enumerating all possible combination for the solution and checking whether each combination satisfies the problem's statement [16]. Our focus is to get the best combination of tourism cost and required time to travel and to get all possible combination we choose a pretrial part of brute force algorithm and DFS. And with the combination of both, we proposed out algorithms which provide our expected output.

\section{Proposed Methodology}

We cast the problem of visiting places as a graph with a group of nodes that connected to its neighbor node and consider path cost as its weight.

Our first algorithm finds all possible path to travel all node at least one time as well as calculate the total cost 
of the path. And using same procedure second algorithm find all paths to return to the source place from where the visiting of mode start. Thus, we describe below our algorithms using DFS algorithm and Brute force algorithm method to find the path and return to the source, this procedure repeated as many times as required until its find out the all possible path.

\subsection{Description of the Core Algorithm}

Our algorithm extends to DFS algorithm. It's a deep-down recursive algorithm. At each recursion generate a path and calculate the cost.

At first, we initialize the graph of the place and define the source place from where the recursion will start, define path index and cost as zero. At the beginning, it makes the source node as visited, add source node to the path and add cost to the total cost and increase the path index. Then it checked that all node visited or not, if all node visited then show the path and total cost of that path, if all node not visited, then find all the unvisited neighbor of the source node and iterate with all the node one by one, at the time of iteration unvisited node define as source node. After both operations make the source unvisited and deduct the cost as well as decrease the path index and return to its parent. This procedure will iterate repeatedly as many time as needed to find all path to visit all node from the source node.

The second algorithm works as the same as the first algorithm, iteration condition same. But it checked source node and destination node is same or not, if same then show the path and total cost of path. If not then find all the unvisited neighbor of the source node and iterate with all the node one by one, at the time of iteration unvisited node define as source node. And this will continue to find all path to return to the source.

\subsubsection{Algorithm of the First Approach}

Input: Weight graph G, start node

Output: all possible paths to visit all node from start node and total cost for all path

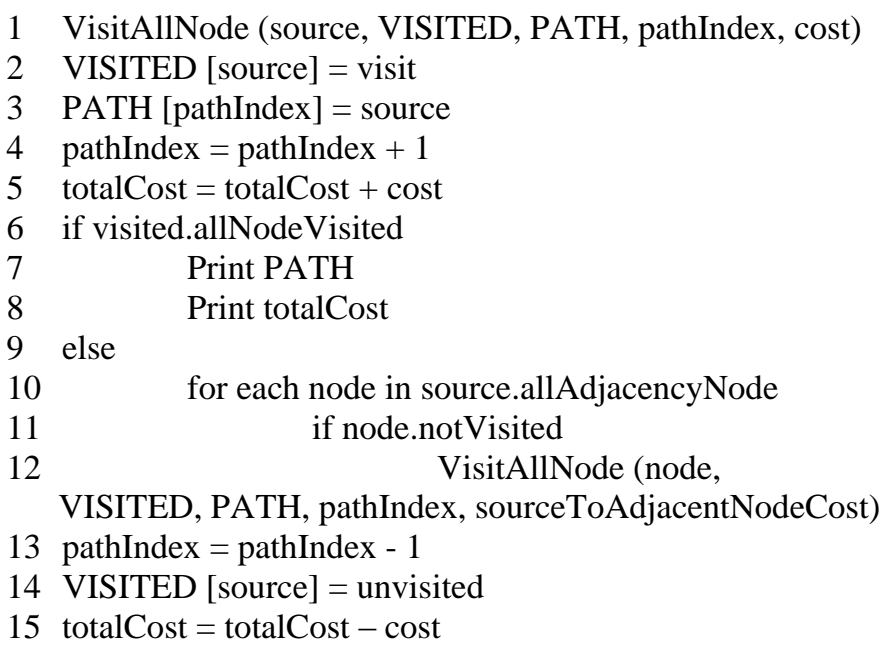

\subsubsection{Algorithm of The Second Approach}

Input: Weight graph $\mathrm{G}=(\mathrm{V}, \mathrm{E}, \mathrm{p})$, source node, destination node.

Output: all possible path to go to destination node from source node and calculate total cost for all path 
1 Algorithm ReturnToSourceNode(source, destination,

VISITED, PATH, pathIndex, cost)

2 VISITED[source] = visit

3 PATH [pathIndex] = source

4 pathIndex $=$ pathIndex +1

5 totalCost $=$ totalCost + cost

6 if source $=$ destination

7 print PATH

$8 \quad$ print totalCost

9 else

10 for each node in source.allAdjacencyNode

11 if node.notVisited

12 ReturnToSourceNode (node, destination, VISITED,

PATH, pathIndex, sourceToAdjacentNodeCost)

13 pathIndex $=$ pathIndex -1

14 VISITED [source] $=$ unvisited

15 totalCost $=$ totalCost - cost

\section{Experiments and Results}

Here we are presenting our algorithms output with experimental data and result.

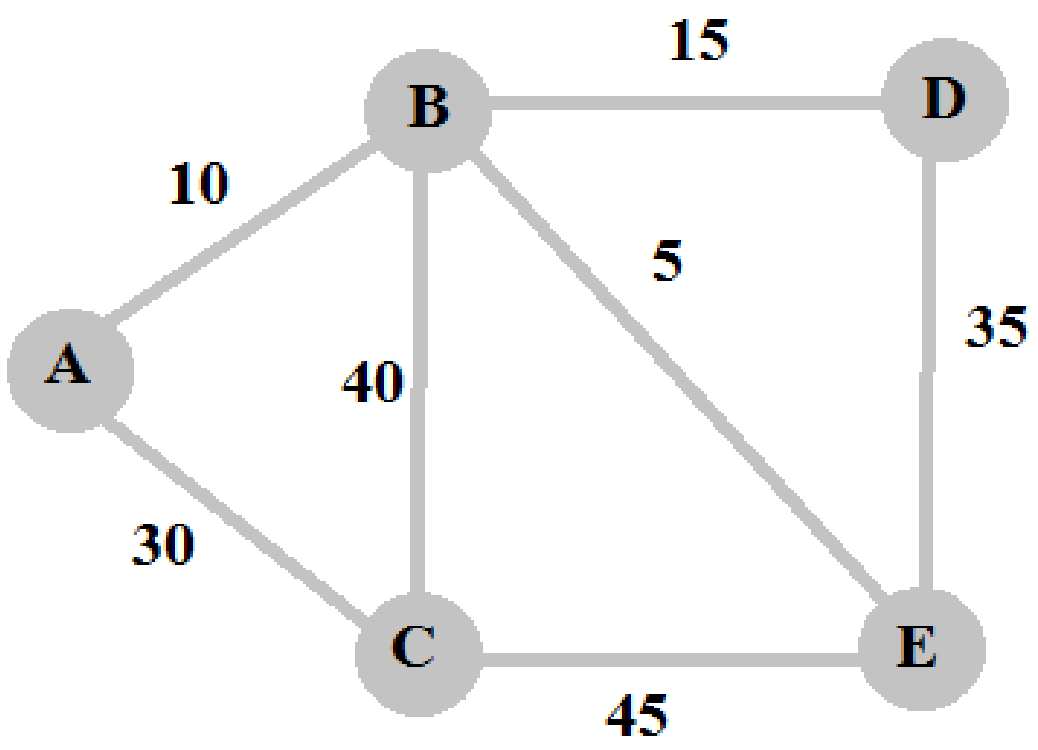

Fig.1. A graph with Five Node and Path Weight

Suppose we have use a graph scenario in Figure 1, for evaluating our approach. In the graph, there are five nodes [A, B, C, D and E]. In this scenario, we take A as start node. Using the first algorithm we find 6 possible paths to visit all node with deferent cost. The result has been shown in Figure 2. 


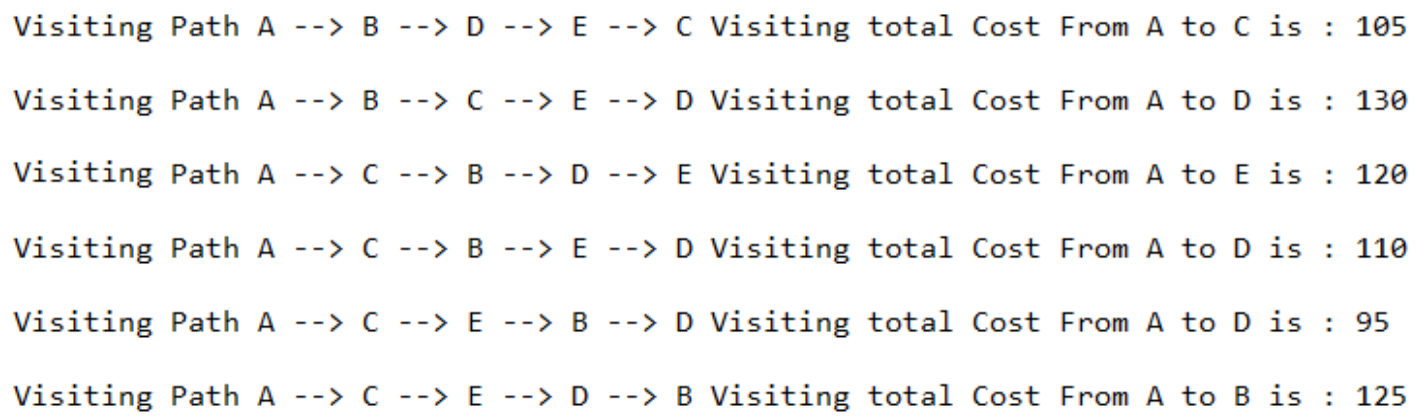

Fig.2. All Path to Visit All Node from A

For the second algorithm, we consider $\mathrm{C}$ as the source node and destination node is A. Here we find 4 possible way to reach A. result shows in Figure 3.

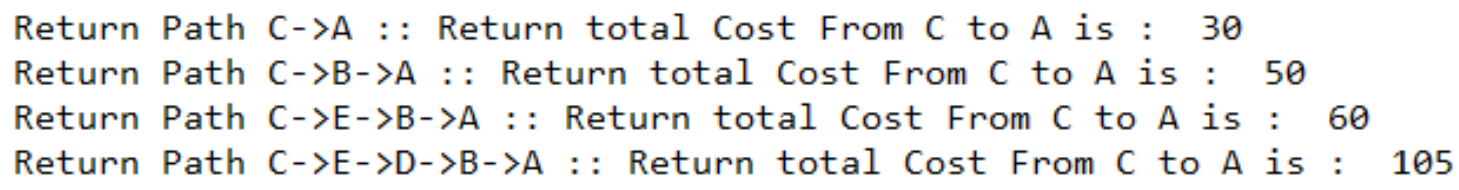

Fig.3. All Path from C to A

If we take source node as D and destination node is A, here we find 7 possible ways to reach A. Result has been shown in Figure 4. Similarly, all return path will be calculated using the procedure of this algorithm.

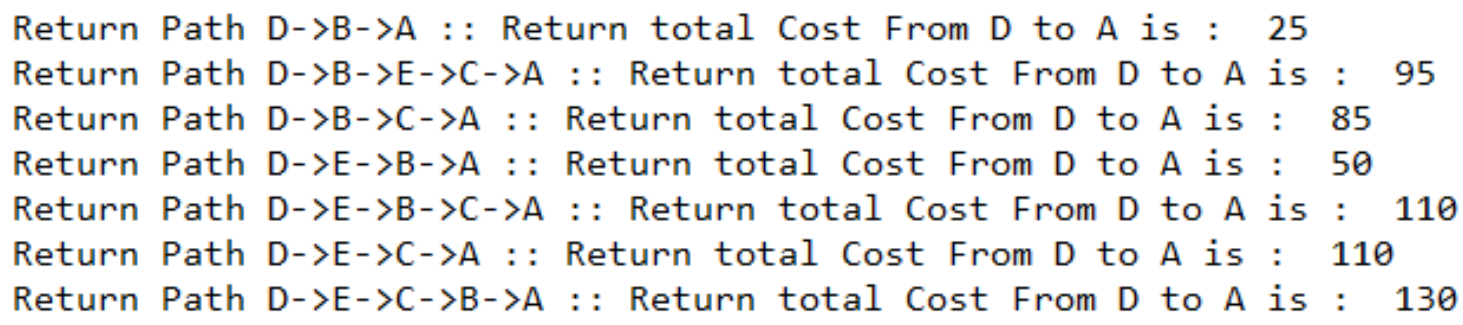

Fig.4. All Path from D to A

Finally, our algorithm will be suggested for visiting path of source to destination and return destination to source. Result has been shown in figure 5.

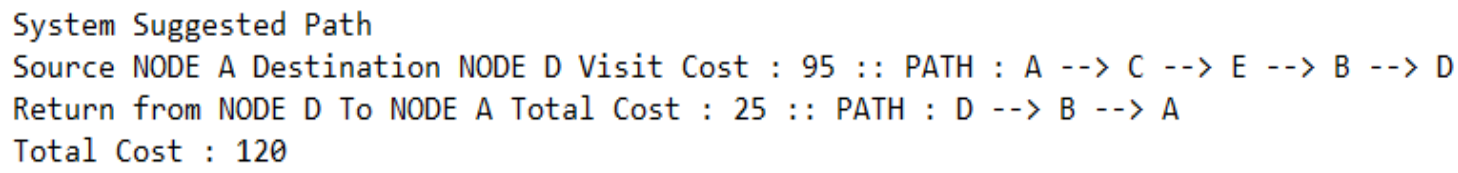

Fig.5. Decision Taking 


\section{Future Work}

We want to implement our Artificial Intelligence and Expert System through a tourism website, which will include all information based on crowd sourced and overall cost of a tour for the desired place. A perfect result will be generated through more data of information, we will suggest best tour place to the user within his/her budget. We will use map API to get proper direction. Our main future goal is to implement our algorithm through a mobile application which automatically takes the user locations using GPS and system will take the suggestion from user also. Transport cost price and hotel cost price will be automatically updated with our API provided to hotel and transport service provider.

\section{Conclusion}

In this paper, we proposed a new method for travel. Contrary to previous work based on the graph traversing we proposed a very efficient method, which extends DFS algorithm. Among the benefits, our approach was to find the maximum results to visit all the place for the tourist to make his/ her own tour plan that which route will be best for his/her. And suggest the best path within given cost.

\section{Limitation}

Our algorithm gives multiple path to visit all node which is achieved, but our algorithm cannot give the lowest total cost to travels all node.

\section{References}

[1] Fatima Leal, Benedita Malheiro and Juan Carlos Burguillo, "Recommendation of Tourism Resources Supported by Crowdsourcing” in DOI: 10.13140/RG.2.2.30159.69283 Conference: International Conference on Information and Communication Technologies in Tourism 2016 (ENTER 2016), At Bilbao, Spain, and Volume: ENTER 2016 PhD Workshop, International Conference on Information and Communication Technologies in Tourism 2016, 18-25.

[2] Jiang, H., 2013. The Research Review of Intelligent Tourism. Journal of Management and Strategy, 4(4), p.65.

[3] Harrill, R., 2004. Residents' attitudes toward tourism development: A literature review with implications for tourism planning. CPL bibliography, 18(3), pp.251-266.

[4] Becken, S. and Wilson, J., 2007. Trip Planning and Decision Making of Self-Drive Tourists- QuasiExperimental Approach. Journal of Travel \& Tourism Marketing, 20(3-4), pp.47-62.

[5] Cohen, S.A., Prayag, G. and Moital, M., 2014. Consumer behaviour in tourism: Concepts, influences and opportunities. Current Issues in Tourism, 17(10), pp.872-909.

[6] Gunn, C.A. with Var, T. (2002) Tourism Planning: basics, concepts and cases, 4th Edition, London: Routledge.

[7] Lai, K., Li, Y. and Feng, X., 2006. Gap between tourism planning and implementation: A case of China. Tourism Management, 27(6), pp.1171-1180.

[8] Buhalis, D., 1998. Strategic use of information technologies in the tourism industry. Tourism management, 19(5), pp.409-421

[9] Baggio, R., 2008. Symptoms of complexity in a tourism system. Tourism Analysis, 13(1), pp.1-20.

[10] Tarjan, R., 1972. Depth-first search and linear graph algorithms. SIAM journal on computing, 1(2), pp.146-160. 
[11] Yuejin, Y., Zhoujun, L. and Huowang, C., 2005. A depth-first search algorithm for mining maximal frequent itemsets [J]. Journal of Computer Research and Development, 3, p.015.

[12] Sharma, M.B., Mandyam, N.K. and Iyangar, S.S., 1989, February. An optimal distributed depth-firstsearch algorithm. In Proceedings of the 17th conference on ACM Annual Computer Science Conference (pp. 287-294). ACM.

[13] Zhou, R. and Hansen, E.A., 2006. Breadth-first heuristic search. Artificial Intelligence, 170(4-5), pp.385-408.

[14] Klöckner, K., Wirschum, N. and Jameson, A., 2004, April. Depth-and breadth-first processing of search result lists. In CHI'04 extended abstracts on Human factors in computing systems (pp. 1539-1539). ACM.

[15] Broumi, S., Bakal, A., Talea, M., Smarandache, F. and Vladareanu, L., 2016, November. Applying Dijkstra algorithm for solving neutrosophic shortest path problem. In Advanced Mechatronic Systems (ICAMechS), 2016 International Conference on (pp. 412-416). IEEE

[16] Aggarwal, C. and Yu, S., 2005. An effective and efficient algorithm for high-dimensional outlier detection. The VLDB Journal-The International Journal on Very Large Data Bases, 14(2), pp.211-221

\section{Authors' Profiles}

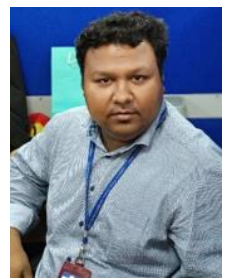

Md. Saef Ullah Miah is currently working as an Assistant Professor in the Computer Sceince department of American International University-Bangladesh (AIUB). Besides teaching, he Possesses real life experience of software development and project management. He has completed MSC and BSC from AIUB. His research interest includes data management, pattern recognition, brain computer interfacing and security in information system design. He serves open source projects and does voluntary works for student and developers community.

Website: http://saefs.xyz

LinkedIn: https://www.linkedin.com/in/mohammad-saef-ullah-miah-08912b71/

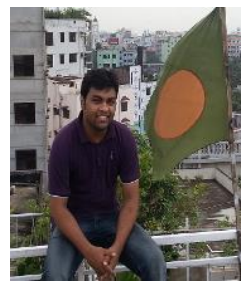

Md. Masuduzzaman is a citizen of Bangladesh and currently working as a lecturer in the dept. of Computer Science at American International University-Bangladesh (AIUB). He has received his Masters degree specialized in Computer Networks and Architecture in 2015 and received his Bachelors degree in Computer Science and Engineering in 2013 from American International University-Bangladesh (AIUB). His areas of interest includes Computer netwoks and architecture, Information and Network Security,Cloud Computing and Internet of Things (IoT). He is also involved in some different sports sector and voluntary work in his country.

LinkedIn Profile: https://www.linkedin.com/in/masud-prince/

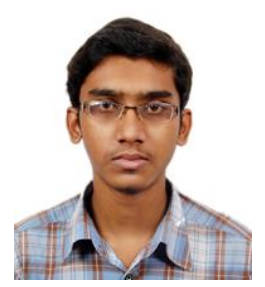

H. M. Mohidul Islam completed his B.Sc. degree in Computer Science \& Engineering (CSE) in 2017 from American International University-Bangladesh (AIUB). Now he is pursuing his Master's degree in Information Technology (IT) at University of Dhaka. At the same time, he is working as a software engineer in ICT Layer (a software company of Bangladesh). His research interest includes data structures, algorithms, machine learning, artificial intelligence, data mining, and bioinformatics.

LinkedIn Profile: https://www.linkedin.com/in/mohidul31/ 

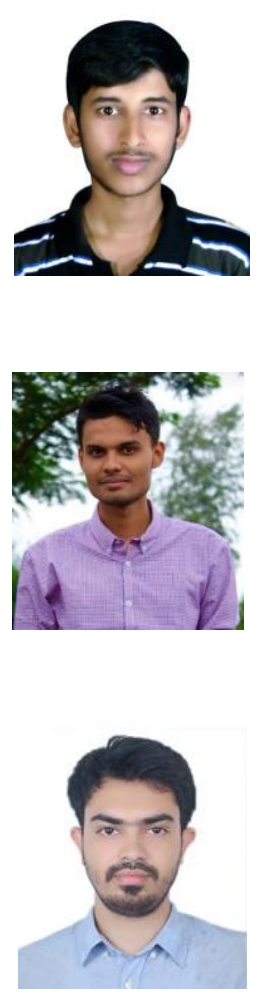

Williyam Sarkar Completed his B.Sc. degree in Computer Science \& Engineering (CSE) in 2017 from American International University-Bangladesh (AIUB). Now he is applying different foreign university to do his post-graduation. At the same time, he is working as a software engineer at Oscillosoft PTY LTD (a software company of Bangladesh). His research interest includes image processing, data structures, algorithms, artificial intelligence and optimization.

LinkedIn Profile: https://www.linkedin.com/in/tonmoy-sarkar-3b41305a

Sajjad Hossain Completed his B.Sc. degree in Computer Science \& Engineering (CSE) in 2017 from American International University-Bangladesh (AIUB). He has completed his Internship at Grameenphone Ltd. Now he is applying different foreign university to complete his masters. His research interest includes pattern recognition, human computer interaction, artificial intelligence and optimization.

LinkedIn Profile: https://www.linkedin.com/in/sajjad-asif-688339109/

Faisal Porag Completed his B.Sc. in Computer Science \& Engineering (CSE) in 2017 from American International University-Bangladesh (AIUB). Now he is pursuing his M.Sc in CSE at Jahangirnagar University, Dhaka. His research interest includes wireless sensor network, image processing, virtual reality and network security.

LinkedIn Profile: https://www.linkedin.com/in/faisal-porag-fpdev

How to cite this paper: Md. Saef Ullah Miah, Md. Masuduzzaman, Williyam Sarkar, H M Mohidul Islam, Faisal Porag, Sajjad Hossain,"Intelligent Tour Planning System using Crowd Sourced Data", International Journal of Education and Management Engineering(IJEME), Vol.8, No.1, pp.22-29, 2018.DOI: 10.5815/ijeme.2018.01.03 